\title{
Borsuk's quasi-equivalence is not transitive
}

\author{
by
Andrzej Kadlof (Warszawa), Nikola Koceić Bilan (Split) and Nikica Uglešić (Split)

\begin{abstract}
Borsuk's quasi-equivalence relation on the class of all compacta is considered. The open problem concerning transitivity of this relation is solved in the negative. Namely, three continua $X, Y$ and $Z$ lying in $\mathbb{R}^{3}$ are constructed such that $X$ is quasiequivalent to $Y$ and $Y$ is quasi-equivalent to $Z$, while $X$ is not quasi-equivalent to $Z$.
\end{abstract}

1. Introduction. In [2] K. Borsuk defined a certain relation on the class $c \mathcal{M}$ of all (metrizable) compacta, called quasi-equivalence and denoted by $\stackrel{q}{\simeq}$. Let us recall its definition (in the original terms of fundamental sequences; see [1]).

Consider any two compacta $X$ and $Y$ lying in AR-spaces $M$ and $N$ respectively, and a neighbourhood $V$ of $Y$ in $N$. Two fundamental sequences $\underline{f}=\left\{f_{k}, X, Y\right\}_{M, N}, \underline{f}^{\prime}=\left\{f_{k}^{\prime}, X, Y\right\}_{M, N}$ are said to be $V$-homotopic (notation: $\underline{f} \widetilde{\bar{V}} \underline{f}^{\prime}$ ) if there exists a neighbourhood $U_{0}$ of $X$ in $M$ such that $f_{k}\left|U_{0} \simeq f_{k}^{\prime}\right| U_{0}$ in $V$ for almost all $k$. (If $V$ is open, then the condition reduces to $f_{k}\left|X \simeq f_{k}^{\prime}\right| X$ in $V$ for almost all $k$.)

Let, in addition, $U$ be a neighbourhood of $X$ in $M$. Then $X$ and $Y$ are said to be $(U, V)$-equivalent in $M, N$ (notation: $X \underset{(U, V)}{\simeq} Y$ ) if there exist two fundamental sequences $\underline{f}=\left\{f_{k}, X, Y\right\}_{M, N}, \underline{g}=\left\{g_{k}, Y, X\right\}_{N, M}$ such that $\underline{g} \underline{f} \underset{U}{\widetilde{\underline{i}}} \underline{i}_{X, M}$ and $\underline{f} \underline{g} \underset{V}{\widetilde{\underline{i}}} \underline{i}_{Y, N}$, where $\underline{i}_{X, M}$ (resp. $\underline{\underline{i}}_{Y, N}$ ) is the fundamental identity sequence for $X$ in $M$ (resp. $Y$ in $N$ ).

Further, Borsuk defined $X$ and $Y$ to be quasi-equivalent in $M, N$ (notation: $X \stackrel{q}{\simeq} Y$ in $M, N)$ if $X \underset{(U, V)}{\simeq} Y$ for every neighbourhood $U$ of $X$ in $M$ and every neighbourhood $V$ of $Y$ in $N$. After proving that the choice of

2000 Mathematics Subject Classification: Primary 54C99; Secondary 55P55.

Key words and phrases: continuum, ANR, inverse sequence, shape, quasi-equivalence, free product of groups. 
the ambient AR-spaces $M$ and $N$ is immaterial, he defined $X$ and $Y$ to be quasi-equivalent (notation: $X \stackrel{q}{\simeq} Y$ ) if $X \stackrel{q}{\simeq} Y$ in some $M, N$.

Borsuk proved that quasi-equivalence is a shape invariant relation and that it is strictly coarser than shape type, i.e.

$$
\begin{aligned}
& (X \stackrel{q}{\simeq} Y) \wedge\left(h(X)=h\left(X^{\prime}\right)\right) \wedge\left(\operatorname{Sh}(Y)=\operatorname{Sh}\left(Y^{\prime}\right)\right) \Rightarrow X^{\prime} \stackrel{q}{\simeq} Y^{\prime} \\
& \operatorname{Sh}(X)=\operatorname{Sh}(Y) \Rightarrow X \stackrel{q}{\simeq} Y ; \\
& (\exists X, Y)(X \stackrel{q}{\simeq} Y) \wedge(\operatorname{Sh}(X) \neq \operatorname{Sh}(Y)) .
\end{aligned}
$$

For instance, all infinite 0-dimensional compacta are quasi-equivalent ([2, Theorem (6.3)]), while their shape types coincide with the topological types ([4, Theorem 20]). Further, in the case of compact ANR's, quasi-equivalence reduces to shape type, and hence to homotopy type. One should also mention that quasi-equivalence preserves some important shape invariants (Betti numbers, movability; [2, Theorems (10.3), (11.1)]). However, it has remained unknown whether quasi-equivalence is indeed an equivalence relation. Specifically, Borsuk stated the following question ([2, Problem (7.13)]): "Is the relation of quasi-equivalence transitive?".

A few months ago, the third named author found by chance an old unpublished manuscript of the first named author, containing a certain example intended to show that quasi-equivalence is not transitive. Unfortunately, an analysis by the second named author showed that the proof was incorrect. However, there was a strong feeling that the example might be appropriate. In this paper we provide a correct proof by using the same example (only the notation is slightly changed).

Thus, Borsuk's quasi-equivalence relation is not transitive because there exist continua $X, Y$ and $Z$, lying in the Euclidian space $\mathbb{R}^{3}$, such that $X$ is quasi-equivalent to $Y$ and $Y$ is quasi-equivalent to $Z$, while $X$ is not quasi-equivalent to $Z$.

2. Preliminaries. The preliminary step in our considerations is to characterize $\stackrel{q}{\simeq}$ in terms of the Mardešić-Segal shape category (see [8]).

Recall the inv-category $\operatorname{HTop}^{\mathbb{N}}$ (see [6]). The objects are all inverse sequences $\boldsymbol{X}=\left(X_{i},\left[p_{i i^{\prime}}\right]\right), \boldsymbol{Y}=\left(Y_{j},\left[q_{j j^{\prime}}\right]\right), \ldots$ of topological spaces with the homotopy classes of mappings as bonding arrows, while the morphisms $\boldsymbol{f}$ : $\boldsymbol{X} \rightarrow \boldsymbol{Y}$ are of the form $\boldsymbol{f}=\left(f,\left[f_{j}\right]\right)$, where $f: \mathbb{N} \rightarrow \mathbb{N}$ and $f_{j}: X_{f(j)} \rightarrow Y_{j}$, $j \in \mathbb{N}$, are such that for every pair $j \leq j^{\prime}$ there exists an $i \geq f(j), f\left(j^{\prime}\right)$ satisfying

$$
\left[f_{j}\right]\left[p_{f(j) i}\right]=\left[q_{j j^{\prime}}\right]\left[f_{j^{\prime}}\right]\left[p_{f\left(j^{\prime}\right) i}\right] .
$$

The composition of $\boldsymbol{f}: \boldsymbol{X} \rightarrow \boldsymbol{Y}$ and $\boldsymbol{g}=\left(g,\left[g_{k}\right]\right): \boldsymbol{Y} \rightarrow \boldsymbol{Z}$ is the morphism

$$
\boldsymbol{h} \equiv \boldsymbol{g} \boldsymbol{f}=\left(f g,\left[g_{k} f_{g(k)}\right]\right): \boldsymbol{X} \rightarrow \boldsymbol{Z},
$$


while the identity morphism on $\boldsymbol{X}$ is $1_{\boldsymbol{X}}=\left(1_{\mathbb{N}},\left[1_{X_{i}}\right]\right)$. With the natural equivalence relation $\boldsymbol{f} \simeq \boldsymbol{f}^{\prime}$, i.e. for every $j$ there exists an $i \geq f(j), f^{\prime}(j)$ such that

$$
\left[f_{j}\right]\left[p_{f(j) i}\right]=\left[f_{j}^{\prime}\right]\left[p_{f^{\prime}(j) i}\right],
$$

one obtains the corresponding quotient category $\operatorname{HTop}^{\mathbb{N}} / \simeq$, i.e. the procategory tow-HTop. The class of a morphism $\boldsymbol{f}$ is denoted by $[\boldsymbol{f}]$. Recall that every class $[\boldsymbol{f}]$ admits a special representative $\boldsymbol{f}^{\prime}$, which means that for every pair $j \leq j^{\prime}$,

$$
\left[f_{j}^{\prime}\right]\left[p_{f^{\prime}(j) f^{\prime}\left(j^{\prime}\right)}\right]=\left[q_{j j^{\prime}}\right]\left[f_{j^{\prime}}^{\prime}\right] .
$$

The quotient (sub)category HcANR $\mathrm{N}^{\mathbb{N}} / \simeq$ is the full subcategory tow-HcANR of tow-HTop (the terms $X_{i}, Y_{j}, \ldots$ of its inverse sequences are compact ANR's). It represents the Mardešić-Segal shape category $\mathcal{S} h$ of compact metrizable spaces (see [6, Chap. I]). Namely,

$$
\mathrm{Ob}(\mathcal{S} h)=\operatorname{Ob}(c \mathcal{M}), \quad \operatorname{Sh}(X, Y) \approx \text { tow-HcANR }(\boldsymbol{X}, \boldsymbol{Y}),
$$

where $\boldsymbol{X}, \boldsymbol{Y}$ are any compact ANR-sequences associated with $X, Y$ respectively, i.e. $\boldsymbol{X}=H \underline{X}$ and $\boldsymbol{Y}=H \underline{Y}$, where $\lim \underline{X}=X$ and $\lim \underline{Y}=Y$, and $H$ denotes the passage from an inverse sequence to the inverse sequence consisting of the same terms and of the homotopy classes of the given bonding mappings. For such a pair $\boldsymbol{X}, \boldsymbol{Y}$, the set tow-HcANR $(\boldsymbol{X}, \boldsymbol{Y})$ represents $\operatorname{Sh}(X, Y)$.

It is a well-known fact ([5]; [1, Chap. IX]) that the Borsuk and MardešićSegal shape theories for compacta are equivalent. The following definitions and facts can be found in [8].

Definition 1. Let $\boldsymbol{f}=\left(f,\left[f_{j}\right]\right), \boldsymbol{f}^{\prime}=\left(f^{\prime},\left[f_{j}^{\prime}\right]\right): \boldsymbol{X} \rightarrow \boldsymbol{Y}$ be morphisms of inverse sequences, and let $s \in \mathbb{N}$. Then $\boldsymbol{f}$ is said to be s-homotopic to $\boldsymbol{f}^{\prime}$, denoted by $\boldsymbol{f} \simeq_{s} \boldsymbol{f}^{\prime}$, provided for every $j \in[1, s]_{\mathbb{N}}$ there exists an $i_{j} \geq$ $f(j), f^{\prime}(j)$ such that

$$
\left[f_{j}\right]\left[p_{f(j) i_{j}}\right]=\left[f_{j}^{\prime}\right]\left[p_{f^{\prime}(j) i_{j}}\right] .
$$

Observe that $\boldsymbol{f} \simeq \boldsymbol{f}^{\prime}$ if and only if $\boldsymbol{f} \simeq_{s} \boldsymbol{f}^{\prime}$ for every $s \in \mathbb{N}$.

LEMma 1.

(i) For every $s \in \mathbb{N}$, the relation $\simeq_{s}$ is an equivalence relation on each set $\operatorname{HTop}^{\mathbb{N}}(\boldsymbol{X}, \boldsymbol{Y})$.

(ii) For every pair $s \leq s^{\prime}, \boldsymbol{f} \simeq_{s^{\prime}} \boldsymbol{f}^{\prime}$ implies $\boldsymbol{f} \simeq_{s} \boldsymbol{f}^{\prime}$. Moreover, for every $s \in \mathbb{N}$, the relation $\simeq_{s}$ is natural from the right in the category HTop $^{\mathbb{N}}$, i.e. for every $\boldsymbol{h}: \boldsymbol{W} \rightarrow \boldsymbol{X}, \boldsymbol{f} \simeq_{s} \boldsymbol{f}^{\prime}$ implies $\boldsymbol{f} \boldsymbol{h} \simeq_{s} \boldsymbol{f}^{\prime} \boldsymbol{h}$. On the other hand, if $\boldsymbol{g}: \boldsymbol{Y} \rightarrow \boldsymbol{Z}$, then $\boldsymbol{f} \simeq_{s} \boldsymbol{f}^{\prime}$ implies $\boldsymbol{g} \boldsymbol{f} \simeq_{t} \boldsymbol{g} \boldsymbol{f}^{\prime}$ whenever $g\left[[1, t]_{\mathbb{N}}\right] \subseteq[1, s]_{\mathbb{N}}$. 
Definition 2. Let $\boldsymbol{X}$ and $\boldsymbol{Y}$ be compact ANR-sequences. Then $\boldsymbol{X}$ is said to be quasi-equivalent to $\boldsymbol{Y}$, denoted by $\boldsymbol{X} \stackrel{q}{\simeq} \boldsymbol{Y}$, provided, for every $n \in \mathbb{N}$, there exist morphisms $\boldsymbol{f}: \boldsymbol{X} \rightarrow \boldsymbol{Y}$ and $\boldsymbol{g}: \boldsymbol{Y} \rightarrow \boldsymbol{X}$ such that $\boldsymbol{g} \boldsymbol{f} \simeq_{n} 1_{\boldsymbol{X}}$ and $\boldsymbol{f g} \simeq_{n} 1_{\boldsymbol{Y}}$.

LEMMA 2. The relation $\stackrel{q}{\simeq}$ is isomorphism (i.e. shape) invariant in towHcANR.

Proof. Let $\boldsymbol{X} \stackrel{q}{\simeq} \boldsymbol{Y}$ and let $\boldsymbol{X} \cong \boldsymbol{X}^{\prime}$ in tow-HcANR. By definition, there exist sequences of maps $\boldsymbol{f}^{n}: \boldsymbol{X} \rightarrow \boldsymbol{Y}$ and $\boldsymbol{g}^{n}: \boldsymbol{Y} \rightarrow \boldsymbol{X}$ satisfying $\boldsymbol{g}^{n} \boldsymbol{f}^{n} \simeq_{n} 1_{\boldsymbol{X}}$ and $\boldsymbol{f}^{n} \boldsymbol{g}^{n} \simeq_{n} 1_{\boldsymbol{Y}}, n \in \mathbb{N}$. Further, there exist morphisms $\boldsymbol{u}: \boldsymbol{X} \rightarrow \boldsymbol{X}^{\prime}$ and $\boldsymbol{v}: \boldsymbol{X}^{\prime} \rightarrow \boldsymbol{X}$ such that $\boldsymbol{v} \boldsymbol{u} \simeq 1_{\boldsymbol{X}}$ and $\boldsymbol{u} \boldsymbol{v} \simeq 1_{\boldsymbol{X}^{\prime}}$. Notice that

$$
(\forall m \in \mathbb{N})\left(\exists s_{m} \geq m\right) \quad u\left[[1, m]_{\mathbb{N}}\right] \subseteq\left[1, s_{m}\right]_{\mathbb{N}} .
$$

For each $m$, let

$$
\boldsymbol{v}^{m} \equiv \boldsymbol{f}^{s_{m}} \boldsymbol{v}: \boldsymbol{X}^{\prime} \rightarrow \boldsymbol{Y} \quad \text { and } \quad \boldsymbol{u}^{m} \equiv \boldsymbol{u} \boldsymbol{g}^{s_{m}}: \boldsymbol{Y} \rightarrow \boldsymbol{X}^{\prime} .
$$

Now, according to Lemma 1 ,

$$
\begin{aligned}
& \boldsymbol{g}^{s_{m}} \boldsymbol{f}^{s_{m}} \simeq_{s_{m}} 1_{\boldsymbol{X}} \Rightarrow \boldsymbol{g}^{s_{m}} \boldsymbol{f}^{s_{m}} \boldsymbol{v} \simeq_{s_{m}} \boldsymbol{v} \Rightarrow \\
& \boldsymbol{u} \boldsymbol{g}^{s_{m}} \boldsymbol{f}^{s_{m}} \boldsymbol{v} \simeq_{m} \boldsymbol{u} \boldsymbol{v} \simeq 1_{\boldsymbol{X}^{\prime}} \Rightarrow \boldsymbol{u}^{m} \boldsymbol{v}^{m} \simeq_{m} 1_{\boldsymbol{X}^{\prime}} \\
& \boldsymbol{v} \boldsymbol{u} \simeq 1_{\boldsymbol{X}} \Rightarrow \boldsymbol{v} \boldsymbol{u} \boldsymbol{g}^{s_{m}} \simeq \boldsymbol{g}^{s_{m}} \Rightarrow \\
& \boldsymbol{f}^{s_{m}} \boldsymbol{v} \boldsymbol{u} \boldsymbol{g}^{s_{m}} \simeq \boldsymbol{f}^{s_{m}} \boldsymbol{g}^{s_{m}} \simeq_{s_{m}} 1_{\boldsymbol{Y}} \Rightarrow \boldsymbol{v}^{m} \boldsymbol{u}^{m} \simeq_{m} 1_{\boldsymbol{Y}} .
\end{aligned}
$$

Thus, $\boldsymbol{X}^{\prime} \stackrel{q}{\simeq} \boldsymbol{Y}$. In the same way one proves that $\boldsymbol{X} \stackrel{q}{\simeq} \boldsymbol{Y}$ and $\boldsymbol{Y} \cong \boldsymbol{Y}^{\prime}$ imply $\boldsymbol{X} \stackrel{q}{\simeq} \boldsymbol{Y}^{\prime}$. Therefore, $\stackrel{q}{\simeq}$ is an isomorphism invariant relation in the category tow-HcANR.

In order to compare Borsuk's quasi-equivalence on compacta to the new relation $\stackrel{q}{\simeq}$ on $\mathrm{Ob}$ (tow-HcANR), we shall prove the following lemma:

Lemma 3. Let $X$ and $Y$ be compacta in the Hilbert cube $Q$, and let $\underline{X}=\left(X_{i}, p_{i i^{\prime}}\right)$ and $\underline{Y}=\left(Y_{j}, q_{j j^{\prime}}\right)$ be any associated inclusion compact ANRsequences respectively. Let $g=\left\{g_{k}, X, Y\right\}$ and $g^{\prime}=\left\{g_{k}^{\prime}, X, Y\right\}$ be fundamental sequences (in $Q)$ and let $\boldsymbol{f}=\left(f,\left[f_{j}\right]\right)$ and $\boldsymbol{f}^{\prime}=\left(f^{\prime},\left[f_{j}^{\prime}\right]\right)$ be morphisms of $\operatorname{HcANR}^{\mathbb{N}}(\boldsymbol{X}, \boldsymbol{Y})$, where $\boldsymbol{X}=H \underline{X}$ and $\boldsymbol{Y}=H \underline{Y}$. If $\underline{g}=\left\{g_{k}, X, Y\right\}$ and $\boldsymbol{f}=\left(f,\left[f_{j}\right]\right)$ as well as $\underline{g}^{\prime}=\left\{g_{k}^{\prime}, X, Y\right\}$ and $\boldsymbol{f}^{\prime}=\left(f^{\prime},\left[f_{j}^{\prime}\right]\right)$ are related, then

(i) for every $n \in \mathbb{N}$ there exists a neighbourhood $V$ of $Y$ in $Q$ such that $\underline{g} \underset{V}{\simeq} \underline{g}^{\prime}$ implies $\boldsymbol{f} \simeq_{n} \boldsymbol{f}^{\prime}$

(ii) for every neighbourhood $V$ of $Y$ in $Q$ there exists an $n \in \mathbb{N}$ such that $\boldsymbol{f} \simeq_{n} \boldsymbol{f}^{\prime}$ implies $\underline{g} \underset{V}{\simeq} \underline{g}^{\prime}$. 
Proof. In this case "to be related" means (see [5] or [1, IX.4])

$$
\begin{aligned}
& f_{j}=g_{f(j)} \mid X_{f(j)}: X_{f(j)} \rightarrow Y_{j}, \quad j \in \mathbb{N}, \\
& g_{i}\left|X_{f(j)} \simeq g_{f(j)}\right| X_{f(j)} \quad \text { in } Y_{j}, i \geq f(j),
\end{aligned}
$$

and, similarly,

$$
\begin{aligned}
& f_{j}^{\prime}=g_{f^{\prime}(j)}^{\prime} \mid X_{f^{\prime}(j)}: X_{f^{\prime}(j)} \rightarrow Y_{j}, \quad j \in \mathbb{N}, \\
& g_{i}^{\prime}\left|X_{f^{\prime}(j)} \simeq g_{f^{\prime}(j)}^{\prime}\right| X_{f^{\prime}(j)} \quad \text { in } Y_{j}, i \geq f^{\prime}(j) .
\end{aligned}
$$

Moreover, we may assume that the index functions $f$ and $f^{\prime}$ are increasing. Now, for (i), if an $n \in \mathbb{N}$ is given, choose $V=Y_{n}$. Then choose a $U_{0} \supseteq X$ in $Q$ coming from $\underline{g} \underset{V}{\simeq} \underline{g}^{\prime}$, and an $i_{0} \in \mathbb{N}$ such that $X_{i_{0}} \subseteq U_{0}$. Let $i_{n}=$ $\max \left\{f(n), f^{\prime}(n), i_{0}\right\}$. By choosing $i_{j}=i_{n}$ for every $j \in[1, n]_{\mathbb{N}}$, the relation $\boldsymbol{f} \simeq_{n} \boldsymbol{f}^{\prime}$ is established. Further, for (ii), if a $V \supseteq Y$ in $Q$ is given, choose the minimal $n \in \mathbb{N}$ such that $Y_{n} \subseteq V$. Let $i_{0} \in \mathbb{N}$ be the maximum of all $i_{j}$ coming from $\boldsymbol{f} \simeq_{n} \boldsymbol{f}^{\prime}$. Then $\underline{g} \underset{V}{\simeq} \underline{g}^{\prime}$ is realized via $U_{0}=X_{i_{0}}$.

Theorem 1. Let $X$ and $Y$ be compacta and let $\boldsymbol{X}$ and $\boldsymbol{Y}$ be compact ANR-sequences associated with $X$ and $Y$ respectively. Then

$$
X \stackrel{q}{\simeq} Y \Leftrightarrow \boldsymbol{X} \stackrel{q}{\simeq} \boldsymbol{Y} .
$$

Consequently, $X$ and $Y$ are quasi-equivalent, $X \stackrel{q}{\simeq} Y$, if and only if, for every $n \in \mathbb{N}$, there exist morphisms $\boldsymbol{f}^{n}: \boldsymbol{X} \rightarrow \boldsymbol{Y}$ and $\boldsymbol{g}^{n}: \boldsymbol{Y} \rightarrow \boldsymbol{X}$ such that $\boldsymbol{g}^{n} \boldsymbol{f}^{n} \simeq_{n} 1_{\boldsymbol{X}}$ and $\boldsymbol{f}^{n} \boldsymbol{g}^{n} \simeq_{n} 1_{\boldsymbol{Y}}$.

Proof. Recall that every compact metrizable space is, up to homeomorphism, the intersection of a decreasing sequence of compact ANR-neighbourhoods in the Hilbert cube. Further, recall (see [5] or [1, IX.4]) that every fundamental sequence $g=\left\{g_{k}, X, Y\right\}$ admits a related morphism $\boldsymbol{f}: \boldsymbol{X} \rightarrow \boldsymbol{Y}$ and vice versa. According to Lemma 3, since Borsuk's quasiequivalence is shape invariant, $X \stackrel{q}{\simeq} Y$ implies that there exist countable families $\left(\boldsymbol{f}^{\left(n, n^{\prime}\right)}\right)$ and $\left(\boldsymbol{g}^{\left(n, n^{\prime}\right)}\right),\left(n, n^{\prime}\right) \in \mathbb{N} \times \mathbb{N}$, of morphisms $\boldsymbol{f}^{\left(n, n^{\prime}\right)}: \boldsymbol{X} \rightarrow \boldsymbol{Y}$ and $\boldsymbol{g}^{\left(n, n^{\prime}\right)}: \boldsymbol{Y} \rightarrow \boldsymbol{X}$ such that

$$
\boldsymbol{g}^{\left(n, n^{\prime}\right)} \boldsymbol{f}^{\left(n, n^{\prime}\right)} \simeq_{n^{\prime}} 1_{\boldsymbol{X}} \text { and } \boldsymbol{f}^{\left(n, n^{\prime}\right)} \boldsymbol{g}^{\left(n, n^{\prime}\right)} \simeq_{n} 1_{\boldsymbol{Y}} .
$$

Clearly, by Lemma 1 , both homotopies hold up to $\min \left\{n, n^{\prime}\right\}$. Thus, by Definition 2 and Lemma 2, the necessity part follows. Conversely, let $\boldsymbol{X} \stackrel{q}{\simeq} \boldsymbol{Y}$, i.e. let there exist morphisms $\boldsymbol{f}^{n}: \boldsymbol{X} \rightarrow \boldsymbol{Y}$ and $\boldsymbol{g}^{n}: \boldsymbol{Y} \rightarrow \boldsymbol{X}, n \in \mathbb{N}$, such that

$$
\boldsymbol{g}^{n} \boldsymbol{f}^{n} \simeq_{n} 1_{\boldsymbol{X}} \text { and } \boldsymbol{f}^{n} \boldsymbol{g}^{n} \simeq_{n} 1_{\boldsymbol{Y}}
$$

Given an ordered pair $\left(n, n^{\prime}\right) \in \mathbb{N} \times \mathbb{N}$, put

$$
\boldsymbol{f}^{\left(n, n^{\prime}\right)}=\boldsymbol{f}^{m} \quad \text { and } \quad \boldsymbol{g}^{\left(n, n^{\prime}\right)}=\boldsymbol{g}^{m}, \quad \text { where } m=\max \left\{n, n^{\prime}\right\} .
$$

Then $X \stackrel{q}{\simeq} Y$ according to Lemmata 3 and 1 . 
REMARK 1. We may assume, without loss of generality, that all the morphisms realizing the relations $\boldsymbol{X} \stackrel{q}{\simeq} \boldsymbol{Y}$ are special with (strictly) increasing index functions. We may also assume that $n^{\prime} \geq n$ implies $f^{n^{\prime}} \geq f^{n}$, and similarly for all other index functions. Further, the conditions $\boldsymbol{g}^{n} \boldsymbol{f}^{n} \simeq_{n} 1_{\boldsymbol{X}}$ etc. may be relaxed to $\boldsymbol{g}^{n} \boldsymbol{f}^{n} \simeq_{s_{n}} 1_{\boldsymbol{X}}$ etc., where $\left(s_{n}\right)$ is an unbounded sequence in $\mathbb{N} \cup\{0\}$.

To end this section we give a useful sufficient condition for a pair of compacta to be quasi-equivalent; it was formulated and proved earlier in the above mentioned manuscript.

Lemma 4. Let $X, Y$ be a pair of compacta satisfying the following condition: For every $\varepsilon>0$ there exist mappings $f: X \rightarrow Y$ and $g: Y \rightarrow X$ such that

$$
(\forall x \in X) d_{X}(g f(x), x)<\varepsilon \quad \text { and } \quad(\forall y \in Y) d_{Y}(f g(y), y)<\varepsilon .
$$

Then $X$ and $Y$ are quasi-equivalent.

Proof. Without loss of generality, we may assume that $X$ and $Y$ lie in the Hilbert cube $Q$. Let $U, V$ be any pair of neighbourhoods of $X, Y$ in $Q$ respectively. There exist compact ANR's $U^{\prime}, V^{\prime}$ such that $X \subseteq U^{\prime} \subseteq \operatorname{Int} U$ and $Y \subseteq V^{\prime} \subseteq$ Int $V$. Let $i: X \hookrightarrow U^{\prime}$ and $j: Y \hookrightarrow V^{\prime}$ be the inclusion mappings. It is well known that there exists an $\varepsilon>0$ such that each pair of $\varepsilon$-near mappings of a metrizable space into $U^{\prime}$ (or into $V^{\prime}$ ) is homotopic. By assumption, there exist mappings $f: X \rightarrow Y$ and $g: Y \rightarrow X$ such that $g f$ and $1_{X}$ as well as $f g$ and $1_{Y}$ are $\varepsilon$-near. Consequently, $g f i, i: X \hookrightarrow U^{\prime}$ as well as $f g j, j: Y \hookrightarrow V^{\prime}$ are $\varepsilon$-near. Therefore, $g f i \simeq i$ and $f g j \simeq j$. This means $g f \simeq 1_{X}$ in $U^{\prime} \subseteq \operatorname{Int} U$ and $f g \simeq 1_{Y}$ in $V^{\prime} \subseteq \operatorname{Int} V$. Let $f=\left\{f_{k}, X, Y\right\}$ and $\underline{g}=\left\{g_{k}, Y, X\right\}$ be fundamental sequences generated by $\tilde{f}$ and $g$ respectively. Now, apply the following fact (mentioned in the introduction):

Let $A$ and $B$ be compacta lying in $Q$, let $W$ be an open neighbourhood of $B$ in $Q$ and let $\underline{h}=\left\{h_{k}, A, B\right\}, \underline{h}^{\prime}=\left\{h_{k}^{\prime}, A, B\right\}$ be fundamental sequences. Then $\underline{h} \underset{W}{\simeq} \underline{h}^{\prime}$ if and only if $h_{k}\left|A \simeq h_{k}^{\prime}\right| A$ in $W$ for almost all $k \in \mathbb{N}$.

Consequently, $\underline{g} \underline{f} \underset{U}{\widetilde{\underline{i}}} \underline{i}_{X}$ and $\underline{f} \underline{g} \underset{V}{\widetilde{\underline{i}}} \underline{i}_{Y}$, where $\underline{i}_{X}$ and $\underline{i}_{Y}$ are the identity fundamental sequences for $X$ in $Q$ and $Y$ in $Q$ respectively. Therefore, $X \stackrel{q}{\simeq} Y$.

3. The example. Let $X$ be an infinite countable one-point union of pointed tori converging to the limit torus. Further, let $Y$ be an infinite countable one-point union of pointed tori converging to the base point. Finally, let $Z$ be the one-point union of $X$ and a pointed circle. An explicit construction is given below. 
For every $k \in \mathbb{N}$, let $A_{k}=S_{k}^{1} \cup \Sigma_{k}^{1} \subseteq \mathbb{R}^{3}$, where $S_{k}^{1}$ and $\Sigma_{k}^{1}$ are the following circles:

$$
\begin{aligned}
& S_{k}^{1}=\left\{(\xi, \eta, 0) \mid\left(\xi-\frac{2 k+3}{2 k+2}\right)^{2}+\eta^{2}=\left(\frac{2 k+3}{2 k+2}\right)^{2}\right\} \\
& \Sigma_{k}^{1}=\left\{(\xi, \eta, 0) \mid\left(\xi-\frac{2 k+3}{2 k+2}\right)^{2}+\eta^{2}=\left(\frac{6 k+1}{8 k+8}\right)^{2}\right\} .
\end{aligned}
$$

Further, let

$$
\begin{aligned}
& S_{\infty}^{1}=\left\{(\xi, \eta, 0) \mid(\xi-1)^{2}+\eta^{2}=1\right\} \subseteq \mathbb{R}^{3}, \\
& \Sigma_{\infty}^{1}=\left\{(\xi, \eta, 0) \mid(\xi-1)^{2}+\eta^{2}=9 / 16\right\} \subseteq \mathbb{R}^{3} .
\end{aligned}
$$

Notice that $A_{k} \cap A_{k^{\prime}}=S_{k}^{1} \cap S_{k^{\prime}}^{1}=\{(0,0,0)\}$ whenever $k \neq k^{\prime} \in \mathbb{N} \cup\{\infty\}$, $\lim \left(S_{k}^{1}\right)=S_{\infty}^{1}$ and $\lim \left(\Sigma_{k}^{1}\right)=\Sigma_{\infty}^{1}$. For every $k \in \mathbb{N} \cup\{\infty\}$, let $T_{k} \subseteq \mathbb{R}^{3}$ be a torus, symmetric with the respect to the $(\xi, \eta)$-plane $\mathbb{R}^{2}$, such that $T_{k} \cap\left(\mathbb{R}^{2} \times\{0\}\right)=A_{k}$. One can easily achieve that $T_{k} \cap T_{k^{\prime}}=\{(0,0,0)\}$ whenever $k \neq k^{\prime} \in \mathbb{N} \cup\{\infty\}$, and $\lim \left(T_{k}\right)=T_{\infty}$. Let

$$
X=\bigcup_{k \in \mathbb{N} \cup\{\infty\}} T_{k} .
$$

Similarly, for every $k \in \mathbb{N}$, let $A_{k}^{\prime}=S_{k}^{\prime 1} \cup \Sigma_{k}^{\prime 1} \subseteq \mathbb{R}^{3}$, where

$$
\begin{aligned}
& S_{k}^{\prime 1}=\left\{(\xi, \eta, 0) \mid\left(\xi-\frac{1}{2^{3 k-3}}\right)^{2}+\eta^{2}=\left(\frac{1}{2^{3 k-3}}\right)^{2}\right\}, \\
& \Sigma_{k}^{\prime 1}=\left\{(\xi, \eta, 0) \mid\left(\xi-\frac{1}{2^{3 k-3}}\right)^{2}+\eta^{2}=\left(\frac{1}{2^{3 k-2}}\right)^{2}\right\} .
\end{aligned}
$$

Notice that $A_{k}^{\prime} \cap A_{k^{\prime}}^{\prime}=S_{k}^{\prime 1} \cap S_{k^{\prime}}^{\prime 1}=\{(0,0,0)\}$ whenever $k \neq k^{\prime} \in \mathbb{N}$, and $\lim \left(S_{k}^{\prime 1}\right)=\lim \left(\Sigma_{k}^{\prime 1}\right)=\{(0,0,0)\}$. For every $k \in \mathbb{N}$, let $T_{k}^{\prime} \subseteq \mathbb{R}^{3}$ be a torus, symmetric with respect to the $(\xi, \eta)$-plane $\mathbb{R}^{2}$, such that $T_{k}^{\prime} \cap\left(\mathbb{R}^{2} \times\{0\}\right)=A_{k}^{\prime}$, $T_{k}^{\prime} \cap T_{k^{\prime}}^{\prime}=\{(0,0,0)\}$ whenever $k \neq k^{\prime}$, and $\lim \left(T_{k}^{\prime}\right)=\{(0,0,0)\}$. Let

$$
Y=\bigcup_{k \in \mathbb{N}} T_{k}^{\prime}
$$

Finally, let

$$
Z=X \cup S^{1}, \quad \text { where } \quad S^{1}=\left\{(\xi, \eta, 0) \mid(\xi+1)^{2}+\eta^{2}=1\right\} .
$$

Clearly, the subspaces $X, Y$ and $Z$ of $\mathbb{R}^{3}$ are compact and path connected.

THEOREM 2. Borsuk's quasi-equivalence relation is not transitive.

Proof. Consider the continua $X, Y$ and $Z$ defined above. It suffices to prove that $X \stackrel{q}{\simeq} Y$ and $Y \stackrel{q}{\simeq} Z$, and that $X$ is not quasi-equivalent to $Z$. Fix $\varepsilon>0$. Then there exists an $n_{\varepsilon}^{\prime} \in \mathbb{N}$ such that $9 /\left(8\left(n_{\varepsilon}^{\prime}+1\right)\right)<\varepsilon$. Given any 
$n^{\prime} \geq \max \left\{8, n_{\varepsilon}^{\prime}\right\}$, set $X_{n^{\prime}}=\bigcup_{k \leq n^{\prime}} T_{k} \subseteq X$, which is a closed subspace. Let $r_{n^{\prime}}: X \rightarrow X_{n^{\prime}}$ be defined by

$$
r_{n^{\prime}}(x)= \begin{cases}x, & x \in X_{n^{\prime}}, \\ \varrho_{n^{\prime}}(x), & x \in X \backslash X_{n^{\prime}}\end{cases}
$$

where $\varrho_{n^{\prime}}: \bigcup_{k>n^{\prime}} T_{k} \rightarrow T_{n^{\prime}}$ is the radial mapping ("blowing up") from the circle passing through the middle of the bounded component of $\mathbb{R}^{3} \backslash T_{\infty}$. Clearly, for every $k \in\left\{n^{\prime}+1, n^{\prime}+2, \ldots\right\} \cup\{\infty\}, \varrho_{n^{\prime}} \mid T_{k}: T_{k} \rightarrow T_{n^{\prime}}$ is a homeomorphism, $\varrho_{n^{\prime}}\left[S_{k}\right]=S_{n^{\prime}}, \varrho_{n^{\prime}}\left[\Sigma_{k}\right]=\Sigma_{n^{\prime}}$ and $\varrho_{n^{\prime}}(0,0,0)=(0,0,0)$. Observe that $r_{n^{\prime}}$ is a retraction. Further, the distance between $r_{n^{\prime}}(x)$ and $x$ reaches its maximum for some $x=(\xi, \eta, \zeta) \in\{(1 / 4,0,0),(7 / 4,0,0),(2,0,0)\} \subseteq A_{0}$. Since

$$
\varrho_{n^{\prime}}\left(\frac{1}{4}, 0,0\right)=\frac{9}{8\left(n^{\prime}+1\right)}, \quad \varrho_{n^{\prime}}\left(\frac{7}{4}, 0,0\right)=\frac{1}{8\left(n^{\prime}+1\right)}, \quad \varrho_{n^{\prime}}(2,0,0)=\frac{1}{n^{\prime}},
$$

and $n^{\prime} \geq 8$, the maximal distance is $9 /\left(8\left(n^{\prime}+1\right)\right)$. Thus, for every $x \in X$,

$$
d\left(r_{n^{\prime}}(x), x\right) \leq \frac{9}{8\left(n^{\prime}+1\right)} \leq \frac{9}{8\left(n_{\varepsilon}^{\prime}+1\right)}<\varepsilon
$$

whenever $n^{\prime} \geq \max \left\{8, n_{\varepsilon}^{\prime}\right\}$.

Similarly, there exists an $n_{\varepsilon}^{\prime \prime} \in \mathbb{N}$ such that $T_{k}^{\prime} \subseteq B((0,0,0), \varepsilon)$ for every $k \geq n_{\varepsilon}^{\prime \prime}$, where $B((0,0,0), \varepsilon)$ is the $\varepsilon$-ball at the origin in $\mathbb{R}^{3}$. Given any $n^{\prime \prime} \geq n_{\varepsilon}^{\prime \prime}$, set $Y_{n^{\prime \prime}}=\bigcup_{k \leq n^{\prime \prime}} T_{k}^{\prime} \subseteq Y$, which is a closed subspace. Let $s_{n^{\prime \prime}}$ : $Y \rightarrow Y_{n^{\prime \prime}}$ be defined by

$$
s_{n^{\prime \prime}}(y)= \begin{cases}y, & y \in Y_{n^{\prime \prime}}, \\ (0,0,0), & y \in Y \backslash Y_{n^{\prime \prime}}\end{cases}
$$

It is obvious that $s_{n^{\prime \prime}}$ is a retraction and that

$$
d\left(s_{n^{\prime \prime}}(y), y\right)<\varepsilon \quad \text { holds for every } y \in Y .
$$

Consider now an $n \geq \max \left\{8, n_{\varepsilon}^{\prime}, n_{\varepsilon}^{\prime \prime}\right\}$ and observe that the subspaces $X_{n}$ and $Y_{n}$ are homeomorphic. Let $h: X_{n} \rightarrow Y_{n}$ be a homeomorphism, and let $r: X \rightarrow X_{n}$ and $s: Y \rightarrow Y_{n}$ be defined as above, i.e. $r=r_{n}$ and $s=s_{n}$. Put

$$
f=j h r: X \rightarrow Y \text { and } g=i h^{-1} s: Y \rightarrow X,
$$

where $i: X_{n} \hookrightarrow X$ and $j: Y_{n} \hookrightarrow Y$ are the inclusion mappings. Let $x \in X$. If $x \in X_{n}$, then $r(x)=x$ and $h r(x)=h(x) \in Y_{n}$, and thus $j h r(x)=h(x)$ and $\operatorname{sjhr}(x)=\operatorname{sh}(x)=h(x)$. Therefore,

$$
g f(x)=i h^{-1} \operatorname{sjh} r(x)=i h^{-1} h(x)=x=r(x) .
$$

If $x \in X \backslash X_{n}$, then $r(x)=\varrho(x)$ and $h r(x)=h \varrho(x) \in Y_{n}$, and thus $j h r(x)=$ $h \varrho(x)$ and $\operatorname{sjhr}(x)=\operatorname{sh} \varrho(x)=h \varrho(x)$. Therefore,

$$
g f(x)=i h^{-1} \operatorname{sjhr}(x)=i h^{-1} h \varrho(x)=i \varrho(x)=\varrho(x)=r(x) .
$$


Consequently,

$$
d(g f(x), x)=d(r(x), x)<\varepsilon \quad \text { for every } x \in X .
$$

In a similar way one can verify that

$$
d(f g(y), y)=d(s(y), y)<\varepsilon \quad \text { for every } y \in Y .
$$

According to Lemma $4, X$ is quasi-equivalent to $Y$.

Let us now prove that $Y$ is quasi-equivalent to $Z$. Fix $\varepsilon>0$. Choose an $n \geq \max \left\{8, n_{\varepsilon}^{\prime}, n_{\varepsilon}^{\prime \prime}\right\}$, where $n_{\varepsilon}^{\prime}$ and $n_{\varepsilon}^{\prime \prime}$ are as in the first part of the proof. Observe that $Y_{n} \cup S_{n+1}^{\prime 1} \subseteq Y$ is a closed subspace. Define $s^{\prime}: Y \rightarrow Y_{n} \cup S_{n+1}^{\prime 1}$ by putting

$$
s^{\prime}(y)= \begin{cases}y, & y \in Y_{n}, \\ \varrho^{\prime}(y), & y \in T_{n+1}^{\prime}, \\ (0,0,0), & y \in Y \backslash Y_{n+1}^{\prime},\end{cases}
$$

where $\varrho^{\prime}: T_{n+1}^{\prime} \rightarrow S_{n+1}^{\prime 1}$ is a retraction of the torus onto the circle. It is clear that $s^{\prime}$ is a retraction satisfying

$$
d\left(s^{\prime}(y), y\right)<\varepsilon \quad \text { for every } y \in Y .
$$

Further, $X_{n} \cup S^{1} \subseteq Z=X \cup S^{1}$ is a closed subspace. Define $r^{\prime}: Z \rightarrow X_{n} \cup S^{1}$ by putting

$$
r^{\prime}(z)= \begin{cases}r(z), & z \in X \\ z, & z \in S^{1}\end{cases}
$$

where $r: X \rightarrow X_{n}$ is the retraction defined in the first part of the proof. Consequently, $r^{\prime}$ is a retraction satisfying

$$
d\left(r^{\prime}(z), z\right)<\varepsilon \quad \text { for every } z \in Z .
$$

Observe that $Y_{n}, S_{n+1}^{1}$ and $Y_{n} \cup S_{n+1}^{1}$ are homeomorphic to $X_{n}, S^{1}$ and $X_{n} \cup S^{1}$ respectively, and that $Y_{n} \cap S_{n+1}^{1}=\{(0,0,0)\}=X_{n} \cap S^{1}$. Let

$$
h^{\prime}: Y_{n} \cup S_{n+1}^{1} \rightarrow X_{n} \cup S^{1}
$$

be a homeomorphism (also on each summand, and keeping $(0,0,0)$ fixed), and let

$$
j^{\prime}: Y_{n} \cup S_{n+1}^{1} \hookrightarrow Y \quad \text { and } \quad i^{\prime}: X_{n} \cup S^{1} \hookrightarrow Z
$$

be the inclusion mappings. Put

$$
f^{\prime}=i^{\prime} h^{\prime} s^{\prime}: Y \rightarrow Z \quad \text { and } \quad g^{\prime}=j^{\prime} h^{\prime-1} r^{\prime}: Z \rightarrow Y .
$$

Let $y \in Y$. If $y \in Y_{n}$, then $s^{\prime}(y)=y$ and $h^{\prime} s^{\prime}(y)=h^{\prime}(y) \in X_{n}$, and thus $i^{\prime} h^{\prime} s^{\prime}(y)=h^{\prime}(y)$ and $r^{\prime} i^{\prime} h^{\prime} s^{\prime}(y)=r^{\prime} h^{\prime}(y)=h^{\prime}(y)$. Therefore,

$$
g^{\prime} f^{\prime}(y)=j^{\prime} h^{\prime-1} r^{\prime} i^{\prime} h^{\prime} s^{\prime}(y)=j^{\prime} h^{\prime-1} h^{\prime}(y)=j^{\prime}(y)=y=s^{\prime}(y) .
$$

If $y \in T_{n+1}^{\prime}$, then $s^{\prime}(y)=\varrho^{\prime}(y) \in S_{n+1}^{1}$ and $h^{\prime} s^{\prime}(y)=h^{\prime} \varrho^{\prime}(y) \in S^{1}$, and thus $i^{\prime} h^{\prime} s^{\prime}(y)=h^{\prime} \varrho^{\prime}(y) \in S^{1}$ and $r^{\prime} i^{\prime} h^{\prime} s^{\prime}(y)=h^{\prime} \varrho^{\prime}(y)$. Therefore,

$$
g^{\prime} f^{\prime}(y)=j^{\prime} h^{\prime-1} r^{\prime} i^{\prime} h^{\prime} s^{\prime}(y)=j^{\prime} h^{\prime-1} h^{\prime} \varrho^{\prime}(y)=j^{\prime} \varrho^{\prime}(y)=\varrho^{\prime}(y)=s^{\prime}(y) .
$$


If $y \in Y \backslash Y_{n+1}$, then $s^{\prime}(y)=(0,0,0)$ and $h^{\prime} s^{\prime}(y)=(0,0,0)$, and thus $i^{\prime} h^{\prime} s^{\prime}(y)=(0,0,0)$ and $r^{\prime} i^{\prime} h^{\prime} s^{\prime}(y)=(0,0,0)$. Therefore,

$$
g^{\prime} f^{\prime}(y)=j^{\prime} h^{\prime-1} r^{\prime} i^{\prime} h^{\prime} s^{\prime}(y)=j^{\prime} h^{\prime-1}(0,0,0)=j(0,0,0)=(0,0,0)=s^{\prime}(y) .
$$

Consequently,

$$
d\left(g^{\prime} f^{\prime}(y), y\right)=d\left(s^{\prime}(y), y\right)<\varepsilon \quad \text { for every } y \in Y .
$$

In a similar way one can verify that

$$
d\left(f^{\prime} g^{\prime}(z), z\right)=d\left(r^{\prime}(z), z\right)<\varepsilon \quad \text { for every } z \in Z .
$$

By Lemma $4, Y$ is quasi-equivalent to $Z$.

It remains to prove that $X$ is not quasi-equivalent to $Z$. For every $i \in \mathbb{N}$ and every $j \in \mathbb{N}$, let $\left(T_{i}^{j}, t_{i}\right)$ be a copy of a pointed torus $(\mathbb{T}, *)$. For every $i \in \mathbb{N}$, let

$$
\left(X_{i}^{\prime}, x_{i}^{\prime}\right)=\left(T_{i}^{1}, t_{i}\right) \vee \cdots \vee\left(T_{i}^{i}, t_{i}\right) .
$$

We may assume that $\left(X_{i+1}^{\prime}, x_{i+1}^{\prime}\right)=\left(X_{i}^{\prime}, x_{i}^{\prime}\right) \vee\left(T_{i+1}^{i+1}, t_{i+1}\right), i \in \mathbb{N}$. Let

$$
p_{i, i+1}: X_{i+1}^{\prime} \rightarrow X_{i}^{\prime}, \quad i \in \mathbb{N},
$$

be defined by requiring that the restrictions

$$
p_{i, i+1} \mid X_{i}^{\prime}: X_{i}^{\prime} \rightarrow X_{i}^{\prime} \quad \text { and } \quad p_{i, i+1} \mid T_{i+1}^{i+1}: T_{i+1}^{i+1} \rightarrow T_{i}^{i} \subseteq X_{i}^{\prime}
$$

be the identities. Notice that $p_{i, i+1}:\left(X_{i+1}^{\prime}, x_{i+1}^{\prime}\right) \rightarrow\left(X_{i}^{\prime}, x_{i}^{\prime}\right)$ is a base point preserving map.

Consider the pointed (compact ANR) inverse sequence $(\underline{X}, \underline{*})=$ $\left(\left(X_{i}^{\prime}, x_{i}^{\prime}\right), p_{i, i+1}\right)$ and its limit $\underline{p}_{*}=\left(p_{i}\right):\left(X^{\prime}, *\right)=\lim (\underline{X}, \underline{*}) \rightarrow(\underline{X}, \underline{*})$. Further, for every $j \in \mathbb{N}$, let

$$
\left(Z_{j}^{\prime}, z_{j}^{\prime}\right)=\left(X_{j}^{\prime}, x_{j}^{\prime}\right) \vee\left(Z_{j}, z_{j}\right)
$$

where $\left(Z_{j}, z_{j}\right)$ is a copy of a pointed circle $\left(\mathbb{S}^{1}, *\right)$. Let

$$
q_{j, j+1}:\left(Z_{j+1}^{\prime}, z_{j+1}^{\prime}\right) \rightarrow\left(Z_{j}^{\prime}, z_{j}^{\prime}\right), \quad j \in \mathbb{N},
$$

be $p_{j, j+1}$ on $X_{j+1}^{\prime}$ and the identity on the copy of $\mathbb{S}^{1}$. Consider the pointed (compact ANR) inverse sequence $(\underline{Z}, \underline{*})=\left(\left(Z_{j}^{\prime}, z_{j}^{\prime}\right), q_{j, j+1}\right)$ and its limit $\underline{q}_{*}=\left(q_{j}\right):\left(Z^{\prime}, *\right)=\lim (\underline{Z}, \underline{*}) \rightarrow(\underline{Z}, \underline{*})$.

Claim. $\left(X^{\prime}, *\right)$ is homeomorphic to $(X,(0,0,0))$, and $\left(Z^{\prime}, *\right)$ is homeomorphic to $(Z,(0,0,0))$.

By construction,

$$
Z=X \cup S^{1},(Z,(0,0,0)) \approx(X,(0,0,0)) \vee\left(\mathbb{S}^{1}, *\right),\left(Z^{\prime}, *\right) \approx\left(X^{\prime}, *\right) \vee\left(\mathbb{S}^{1}, *\right)
$$

Further, all the mappings preserve base points. Thus, it suffices to prove that $X^{\prime} \approx X$. Let $\underline{p}^{\prime}=\left(p_{i}^{\prime}\right): X \rightarrow \underline{X}$ be defined by

$$
p_{i}^{\prime}=h_{i} r_{i}: X \rightarrow X_{i}^{\prime}, \quad i \in \mathbb{N},
$$


where $h_{i}$ is determined by the obvious homeomorphisms on the corresponding tori. Then $p^{\prime}$ distinguishes points and every $p_{i}^{\prime}$ is surjective. By applying Theorem 6 of $\left[\overline{6}\right.$, I.5.2], we infer that $p^{\prime}: X \rightarrow \underline{X}$ is the limit. Therefore, $X$ and $X^{\prime}$ are homeomorphic.

Set

$$
H \underline{X}=\left(X_{i}^{\prime},\left[p_{i, i+1}\right]\right) \equiv \boldsymbol{X} \quad \text { and } \quad H \underline{Z}=\left(Z_{j}^{\prime},\left[q_{j, j+1}\right]\right) \equiv \boldsymbol{Z} .
$$

According to Theorem 1, it remains to prove that $\boldsymbol{X}$ is not quasi-equivalent to $\boldsymbol{Z}$. Suppose, on the contrary, that $\boldsymbol{X} \stackrel{q}{\simeq} \boldsymbol{Z}$. Then, by Definition 2 and Remark 1 , for every $n \in \mathbb{N}$, there exist special morphisms $\boldsymbol{f}^{n}: \boldsymbol{X} \rightarrow \boldsymbol{Z}$ and $\boldsymbol{g}^{n}: \boldsymbol{Z} \rightarrow \boldsymbol{X}$ such that $\boldsymbol{g}^{n} \boldsymbol{f}^{n} \simeq_{n} 1_{\boldsymbol{X}}$ and $\boldsymbol{f}^{n} \boldsymbol{g}^{n} \simeq_{n} 1_{\boldsymbol{Z}}$. Let $n=1$, and write $\boldsymbol{f}^{1} \equiv \boldsymbol{f}=\left(f,\left[f_{j}\right]\right)$ and $\boldsymbol{g}^{1} \equiv \boldsymbol{g}=\left(g,\left[g_{i}\right]\right)$. Since all $X_{i}^{\prime}$ and $Z_{j}^{\prime}$ are ANR-continua, one may assume that all the mappings $f_{j}$ and $g_{i}$ preserve the base points. Since $\boldsymbol{g} \boldsymbol{f} \simeq_{1} 1_{\boldsymbol{X}}$, the diagram

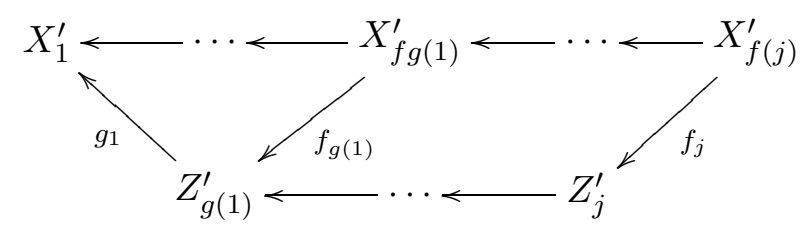

commutes up to homotopy. Let us apply the fundamental group functor $\pi_{1}$ to the left triangle of $(\star)$ (the choice of base points is irrelevant):

$$
\pi_{1}\left(T_{1}^{1}\right) \leftarrow \pi_{g_{1 \#}} \pi_{\pi_{g(1) \#}\left(T_{f g(1)}^{1}\right) * \cdots * \pi_{1}\left(T_{f g(1)}^{f g(1)}\right)} \pi_{\left.\pi_{g(1)}\right) * \cdots * \pi_{1}\left(T_{g(1)}^{g(1)}\right) * \pi_{1}\left(S^{1}\right)}
$$

Recall that the fundamental group of a finite wedge is the corresponding free product (by van Kampen's theorem, [3, Theorem 3.1, p. 122]), and that the fundamental groups of a circle and of a torus are $\mathbb{Z}$ and $\mathbb{Z} \times \mathbb{Z} \equiv \mathbb{Z}^{2}$ respectively. Observe that $f_{g(1) \#} \mid \pi_{1}\left(T_{f g(1)}^{i}\right)$ is a monomorphism of $\mathbb{Z}^{2}$ into $\mathbb{Z}^{2} * \cdots * \mathbb{Z}^{2} * \mathbb{Z}$, for every $i=1, \ldots, f g(1)$. Thus,

$$
\mathbb{Z}^{2} \cong\left(f_{g(1) \#} \mid \pi_{1}\left(T_{f g(1)}^{i}\right)\right)\left(\mathbb{Z}^{2}\right) \leq \mathbb{Z}^{2} * \cdots * \mathbb{Z}^{2} * \mathbb{Z} .
$$

From the Kurosh subgroup theorem ([7, Theorem 1.10, p. 178]) it follows that if $H \leq G=G_{1} * \cdots * G_{n}$, then $H \cong F * H_{1}^{\sigma_{1}} * \cdots * H_{n}^{\sigma_{n}}$, where every $H_{i}$ is a subgroup of some $G_{j}$, every $\sigma_{i} \in G$ and $F$ is a free group.

Recall also that $\mathbb{Z}^{2}$ is not decomposable into a free product (see [7, Proposition 15.14 , p. 107]). Since $\mathbb{Z}^{2}$ is not a free group, the Kurosh subgroup theorem implies that $\mathbb{Z}^{2} \cong\left(f_{g(1) \#} \mid \pi_{1}\left(T_{f g(1)}^{i}\right)\right)\left(\mathbb{Z}^{2}\right) \cong H_{i}^{\sigma_{i}}, H_{i} \leq \pi_{1}\left(T_{g(1)}^{j}\right) \cong \mathbb{Z}^{2}$, for some $j \in\{1, \ldots, g(1)\}$, and $\sigma_{i} \in \pi_{1}\left(T_{g(1)}^{1}\right) * \cdots * \pi_{1}\left(T_{g(1)}^{g(1)}\right) * \pi_{1}\left(S^{1}\right) \cong$ 
$\mathbb{Z}^{2} * \cdots * \mathbb{Z}^{2} * \mathbb{Z}$. Let $a \in \pi_{1}\left(T_{f g(1)}^{i}\right), i \in\{1, \ldots, f g(1)\}$. Then

$$
f_{g(1) \#}(a)=\sigma b \sigma^{-1}, \quad \sigma \in \mathbb{Z}^{2} * \cdots * \mathbb{Z}^{2} * \mathbb{Z}, b \in \pi_{1}\left(T_{g(1)}^{j}\right),
$$

for some $j \in\{1, \ldots, g(1)\}$. Since $f_{g(1) \#} \mid \pi_{1}\left(T_{f g(1)}^{i}\right)$ is a monomorphism, its image in $\mathbb{Z}^{2} * \cdots * \mathbb{Z}^{2} * \mathbb{Z}$ must be isomorphic to $\pi_{1}\left(T_{g(1)}^{j}\right) \cong \mathbb{Z}^{2}$ for some $j \in\{1, \ldots, g(1)\}$. Consequently, if $a_{1} \cdots a_{m} \in \pi_{1}\left(T_{f g(1)}^{1}\right) * \cdots * \pi_{1}\left(T_{f g(1)}^{f g(1)}\right)$, where $a_{k} \in \pi_{1}\left(T_{f g(1)}^{i_{k}}\right)$, then

$$
f_{g(1) \#}\left(a_{1} \cdots a_{m}\right)=\sigma_{1} b_{1} \sigma_{1}^{-1} \cdots \sigma_{m} b_{m} \sigma_{m}^{-1},
$$

for some $\sigma_{k} \in \mathbb{Z}^{2} * \cdots * \mathbb{Z}^{2} * \mathbb{Z}$ and $b_{k} \in \pi_{1}\left(T_{g(1)}^{j_{k}}\right), k=1, \ldots, m, j_{k} \in$ $\{1, \ldots, g(1)\}$.

Further, the right rectangle of $(\star)$ yields the commutative diagram

$$
\begin{gathered}
\pi_{1}\left(T_{f g(1)}^{1}\right) * \cdots * \pi_{1}\left(T_{f g(1)}^{f g(1)}\right) \longleftarrow \\
\downarrow_{g(1) \#}\left(T_{f(j)}^{1}\right) * \cdots * \pi_{1}\left(T_{f(j)}^{f(j)}\right) \\
\pi_{1}\left(T_{g(1)}^{1}\right) * \cdots * \pi_{1}\left(T_{g(1)}^{g(1)}\right) * \pi_{1}\left(S^{1}\right) \longleftarrow \\
f_{j \#} \\
\pi_{1}\left(T_{j}^{1}\right) * \cdots * \pi_{1}\left(T_{j}^{j}\right) * \pi_{1}\left(S^{1}\right)
\end{gathered}
$$

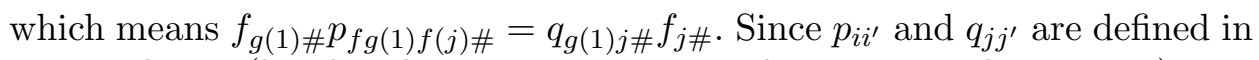
a special way (by the identity mappings on the corresponding copies), one readily sees that, for every $j \geq g(1)$, the restriction $f_{j \#} \mid \pi_{1}\left(T_{f(j)}^{i}\right)$ is also a monomorphism. Therefore, by following the same arguments, one can find that $f_{j \#}$ acts via a formula analogous to that for $f_{g(1) \#}$.

Consider now the relation $\boldsymbol{f g} \simeq_{1} 1_{\boldsymbol{Z}}$ inducing the retraction

$$
r: Z_{1}^{\prime}=T_{1}^{1} \vee S^{1} \rightarrow S^{1}, \quad r\left[T_{1}^{1}\right]=\{*\},
$$

i.e. the following diagram:

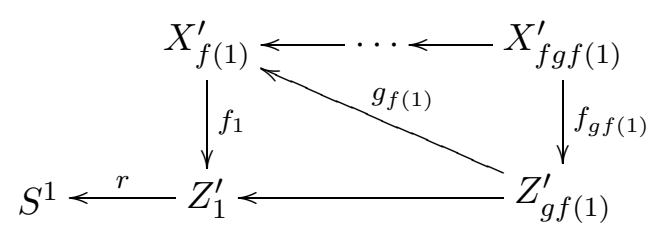

(Caution: The right triangle might not homotopy commute, though the rectangle and the left triangle must homotopy commute!) Applying $\pi_{1}$ to the left triangle and to the rectangle yields the commutative diagrams

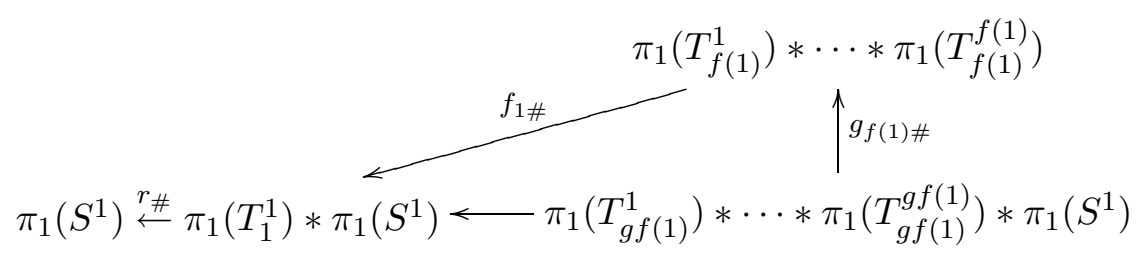




$$
\begin{aligned}
& \pi_{1}\left(T_{f(1)}^{1}\right) * \cdots * \pi_{1}\left(T_{f(1)}^{f(1)}\right) \longleftarrow \pi_{1}\left(T_{f g f(1)}^{1}\right) * \cdots * \pi_{1}\left(T_{f g f(1)}^{f g f(1)}\right)
\end{aligned}
$$

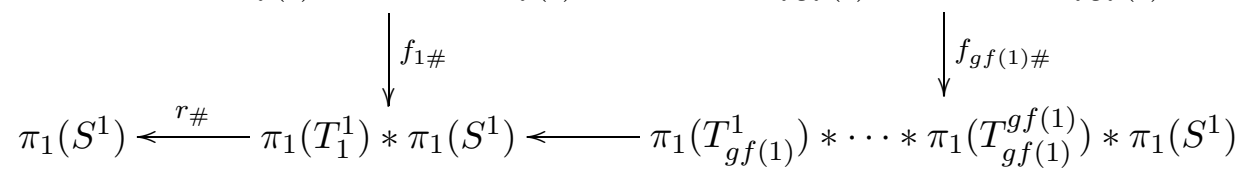

Now, the composition

$$
r_{\#} f_{1 \#} g_{f(1) \#}: \mathbb{Z}^{2} * \cdots * \mathbb{Z}^{2} * \mathbb{Z} \rightarrow \mathbb{Z}
$$

is the trivial homomorphism because $r_{\#} f_{1 \#}$ is trivial. Namely, the restrictions of the bonding homomorphisms are the identities on the corresponding copies, $f_{1 \#} p_{f(1) f g f(1) \#}=q_{1 g f(1) \#} f_{g f(1) \#}, g f(1) \geq g(1)$ and we have already proved how $f_{g f(1) \#}$ acts. Thus, for every $a \in \pi_{1}\left(T_{f(1)}^{i}\right), i \in\{1, \ldots, f(1)\}$, we have $f_{1 \#}(a)=\sigma b \sigma^{-1}$ for some $b \in \pi_{1}\left(T_{1}^{1}\right), \sigma \in \pi_{1}\left(T_{1}^{1}\right) * \pi_{1}\left(S^{1}\right)$. Therefore, $r_{\#} f_{1 \#}(a)=r_{\#}\left(\sigma b \sigma^{-1}\right)=r_{\#}(\sigma) r_{\#}\left(\sigma^{-1}\right)$, and hence $r_{\#} f_{1 \#}$ must be trivial. On the other hand, by the definitions of the relevant mappings, the composition

$$
r_{\#} q_{1 g f(1) \#}: \mathbb{Z}^{2} * \cdots * \mathbb{Z}^{2} * \mathbb{Z} \rightarrow \mathbb{Z}
$$

preserves the free factor $\pi_{1}\left(S^{1}\right) \cong \mathbb{Z}$, so it is not trivial. Therefore, the two displayed compositions cannot be equal.

\section{References}

[1] K. Borsuk, Theory of Shape, Monografie Mat. 59, Polish Sci. Publ., Warszawa, 1975.

[2] - Some quantitative properties of shapes, Fund. Math. 93 (1976), 197-212.

[3] R. C. Lyndon and P. E. Schupp, Combinatorial Group Theory, Springer, Berlin, 1977.

[4] S. Mardešić and J. Segal, Shapes of compacta and ANR-systems, Fund. Math. 72 (1971), 41-59.

[5] - - - Equivalence of the Borsuk and the ANR-system approach to shape, ibid. 72 (1971), 61-78.

[6] - - - Shape Theory, North-Holland, Amsterdam, 1982.

[7] W. S. Massey, Algebraic Topology: An Introduction, Springer, New York, 1977.

[8] N. Uglešić, A note on the Borsuk quasi-equivalence, submitted.

Warsaw University, Poland

E-mail: akadlof@mks.com.pl
Department of Mathematics

University of Split Teslina 12/III, 21000 Split, Croatia E-mail: koceic@pmfst.hr uglesic@pmfst.hr

Received 12 September 2005;

in revised form 26 October 2007 